\title{
Precentral gyrus abnormal connectivity in male and female patients with schizophrenia
}

\author{
Mahdi Zarei \\ Department of Bioengineering, School of Pharmacy, University of California San Francisco, San Francisco, CA 94158, USA. \\ Correspondence to: Dr. Mahdi Zarei, Department of Bioengineering, School of Pharmacy, University of California San Francisco, \\ 1550 4th Street, Bldg 19B, San Francisco, CA 94158, USA. E-mail: mahdi.zarei@ucsf.edu, mehdi.nn@gmail.com
}

How to cite this article: Zarei M. Precentral gyrus abnormal connectivity in male and female patients with schizophrenia. Neuroimmuno/ Neuroinflammation 2018;5:13. http://dx.doi.org/10.20517/2347-8659.2018.02

Received: 8 Jan 2018 First Decision: 8 Feb 2018 Revised: 27 Mar 2018 Accepted: 29 Mar 2018 Published: 23 Apr 2018

Science Editor: Athanassios P. Kyritsis Copy Editor: Guang-Zhe Zhu Production Editor: Cai-Hong Wang

\begin{abstract}
Aim: Dysfunction of the precentral gyrus plays a role in the impairments of voluntary movement associated with schizophrenia and it has significantly reduced functional activity in patients with schizophrenia. The aim of this study was to demonstrate the precentral gyrus alteration and its abnormal connectivity in schizophrenia.
\end{abstract}

Methods: The region of interest-based analysis method was used to investigate the precentral gyrus connectivity alteration in schizophrenia. The resting-state functional magnetic resonance imaging data of healthy control subjects and patients with schizophrenia (Centers of Biomedical Research Excellence data set) was used to examine the aberrant functional brain connectome in schizophrenia. This data set contains raw anatomical and functional magnetic resonance data from 72 patients with schizophrenia and 75 healthy controls, ranging in age from 18 to 65 years old.

Results: Our results show precentral gyrus has abnormal communication with thalamus, hippocampus, parahippocampal gyrus, posterior division of supramarginal gyrus and medial prefrontal cortex ( $P F D R=0.05$ ). This information is expected to provide a better understanding of altered functional connectivity of the precentral gyrus in the male and female patients with schizophrenia.

Conclusion: Collectively, these findings support the hypothesis that precentral gyrus has an abnormal connectivity in schizophrenia and this alteration is not the same in the male and female patients with schizophrenia.

Keywords: Schizophrenia, functional magnetic resonance imaging, functional connectivity, resting-state, precentral gyrus

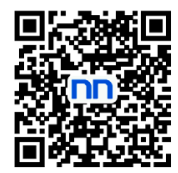




\section{INTRODUCTION}

Schizophrenia is a severe mental disorder, affecting $\sim 1 \%$ of the population ${ }^{[1,2]}$. Recent studies have shown dysfunction of the precentral gyrus (PreCG) has long been thought to play a role in the impairments of voluntary movement associated with Schizophrenia and it has significantly reduced functional activity (FC) in patients with schizophrenia ${ }^{[3,4]}$. Also, schizophrenia is associated with volume deficits in PreCG ${ }^{[5]}$. Another research indicates the patients with schizophrenia showed lower activation in left PreCG than right PreCG $^{[6]}$ and a regional homogeneity $(\mathrm{ReHo})$ study showed decreased ReHo in right precentral gyrus ${ }^{[7]}$. In this research, we examined the PreCG functional connectivity impairment in patients with schizophrenia using ROI based analysis ${ }^{[8]}$. We used the Center for Biomedical Research Excellence data set (COBRE) ${ }^{[9]}$ to demonstrate how the functional connectivity of the PreCG with the rest of the brain regions changes in schizophrenia. In conjunction with previous studies ${ }^{[10-13]}$, our results indicate the PreCG has abnormal connectivity with brain regions like Thalamus and Hippocampus, but these impairments are not similar in two hemispheres. We also analyzed the functional connectivity differences between male and female patients with schizophrenia and showed the regions like Thalamus are more affected in the female patients with schizophrenia.

\section{METHODS}

\section{Data}

Two groups of subjects from the COBRE data set are used to examine the aberrant functional brain connectome in schizophrenia. COBRE data set contains raw anatomical and functional MRI data from patients with schizophrenia and healthy controls. Some papers that by the COBRE group published on this data ${ }^{[14-16]}$. This data set is available on and contains raw anatomical and functional MR data from patients with Schizophrenia and healthy controls, ranging in age from 18 to 65 years old. Resting fMRI, anatomical MRI, phenotype data for every participant including gender, age, handedness and diagnostic information are released ${ }^{[17]}$. More details about the COBRE data set can be found in the Table 1.

\section{Analysis}

Different preprocessing methods like realignment ${ }^{[18]}$, coregistering, normalization are applied to the structural and functional data. The functional volumes are coregistered with the region of interest and structural volumes ${ }^{[19,20]}$. Regions of interest and all the Brodmann areas defined by the Talairach daemon assigned to all subjects. By segmentation of structural image for each subject, grey matter, white matter and cerebrospinal fluid (CSF) mask generated. Here, the time series of interest are numbers of PCA components.

\section{First and second level of covariates}

In this step, the realignment parameters in Blood-Oxygen-Level-Dependent (BOLD) model is defined (the first level covariate), then in the second level covariate, the group level regressor is performed. We categorized the data input data into 4 groups: females and males healthy control, females and males patients with schizophrenia. After defining the experimental data, the functional data is imported, then the structural data segmented to define the grey matter, white matter, the cerebrospinal fluid region of interest. By performing principal component analysis (PCA) on within region of interests the ROIs time series is extracted.

\section{Data preprocessing}

Before analyzing the data, we need to explore and remove the confound. The different source of possible confounds like cerebrospinal fluid and white matter signal and within-subject covariate (realignment parameters) are considered. We chose the 5-dimension numbers of temporal components are being used. Similarly, numbers of dimension for the white matter was 5 and the derivative order was 0 and the histogram 
Table 1. Demographic characteristics of subjects in COBRE data

\begin{tabular}{lccc}
\hline & N & Age (SD) & Female (\%) \\
\hline Patients with schizophrenia & 72 & $38.16(13.89)$ & 0.19 \\
Healthy control & 74 & $35.82(11.58)$ & 0.31 \\
\hline
\end{tabular}

\section{Connectivity histogram before denoising}

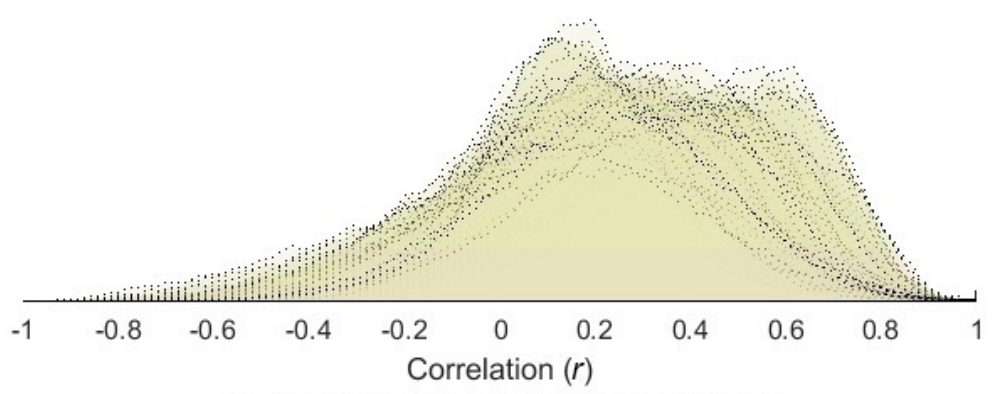

Connectivity histogram after denoising

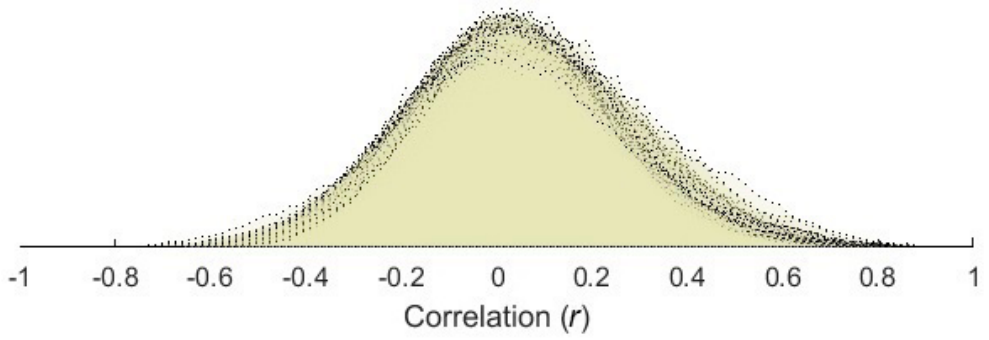

Figure 1. Connectivity histogram before and after denoising

plot $r$ value before and after confounds removal and the band-pass filter is set to $(0.008,0.09)$. Here the histograms $r$-square to identify outlier subject and the quality control is computed for every subject. Figure 1 shows the connectivity histogram before and after denoising.

The functional connectivity (CONN) and Statistical Parametric Mapping (SPM) toolboxes ${ }^{[21]}$ used for spatially preprocessing (realignment, coregistering, normalization) of structural and functional data of functional data are done using SPM toolbox. The functional volumes are coregistered with the region of interest and structural volumes. ROI-to-ROI correlational analysis was carried out by CONN toolbox and SPM8. The preprocessing of the functional images considered of band-pass filtering of 0.008-0.09 Hz, motion correction, registration to structural images and spatial normalization to the MNI template. Then to reduce the physiological noise source, a Component-Based Noise Correction Method (CompCor) has been used ${ }^{[22]}$. CompCor can be used for the reduction of noise in both blood oxygenation level dependent and perfusionbased functional magnetic resonance imaging data. False discovery rate correction is used for multiple hypothesis testing. Number of PCA components to be extracted for each ROI is set to one. It means the time-series of interest is defined as the average BOLD activation within the ROI voxels, but it's possible to define it as the principal eigenvariates of the time-series within the ROI voxels. Regions of interest and all the Brodmann areas defined by Talairach daemon assigned to all subjects. By segmentation of structural image for each subject, grey matter, white matter, and CSF masks were generated. Bivariate correlation is used as a functional connectivity measure between two areas. General linear model (GLM) ${ }^{[23]}$ used for comparison of connectivity results between genders and between control and Schizophrenia patients. 
Table 2. The connectivity contrast values between the PerCG right and the targets $(P=0.05)$

(the complete list of the effects size is provided in the supplementary data)

\begin{tabular}{llll}
\hline Analysis Unit & \multicolumn{1}{c}{ Statistic } & p-unc & p-FDR \\
\hline Seed intensity = 18.77, size $=5$ & PreCG r & $\mathrm{F}(30)(113)=3.17$ & 0.0000 \\
PreCG r - Hippocampus I & $\mathrm{T}(142)=4.16$ & 0.0001 & 0.0074 \\
PreCG r -Thalamus I & $\mathrm{T}(142)=-3.92$ & 0.0001 & 0.0093 \\
PreCG r - pPaHC I & $\mathrm{T}(142)=3.62$ & 0.0004 & 0.0151 \\
PreCG r -Thalamus r & $\mathrm{T}(142)=-3.54$ & 0.0005 & 0.0151 \\
PreCG r - - pSMG r & $\mathrm{T}(142)=-3.53$ & 0.0006 & 0.0151 \\
\hline
\end{tabular}

\section{ROI based analysis}

We investigated the hypothesis of connectivity difference in schizophrenia and used ROI analyzing for the PreCG region of the brain. Two-sample $t$-test analyzes computed via SPM ${ }^{[24]}$ to compare the connectivity results of patients $v s$. controls and male patient $v s$. women patient to compare the connectivity across two groups. Connectivity values (Fisher-transformed correlation coefficients) between the seed and the identified ROI was extracted from the connectivity map. The different source of interest can be defined for analyzing.

\section{First level voxel-based analysis}

For a subject or condition, it is possible to perform voxel-to-voxel analyzing that applies the matrix of voxelto-voxel connectivity values and there is no need for the prior region of interest or seed analysis. In this method, we can investigate whole-brain connectivity. The voxel-based analyzing can be on connectivity pattern (principal component analysis) between a voxel and the rest of the brain (MVPA). Another voxelbased measure is available in CONN toolboxes is indexed that calculates the average local connectivity between each voxel and its neighbors (integrated local correlation) ${ }^{[25]}$ or instead of average, the spatial asymmetry of the local connectivity can be used (radial correlation contrast) ${ }^{[26]}$. Also, instead of local connectivity, global connectivity pattern between a voxel and the rest of the brain can be used (e.g., radial similarity contrast $)^{[27]}$. More details about measuring the index can be found in ${ }^{[28]}$.

\section{Second level analysis}

In the second level analysis step, the between-subject contrast can be considered (e.g., to compare different groups like male $v s$. female to see main effects in the connectivity within each group). In the ROI-to-ROI analyze, the first-level connectivity-measure matrix is used and the results can be a threshold at the desired $P$-value threshold. In this step by graph theoretical analyzing method provides the network measures like efficiency, centrality, and cost/degree to test the between-subject contrast.

\section{RESULTS}

The connectivity contrast values between the PreCG right and the targets show the PreCG has abnormal communication with thalamus, hippocampus, pPaHC, pSMG and mPFC $(P=0.05)$. Figure 2 shows ROI-level results for PreCG left and right PreCG. Effects size for each region is shown by the dot size. We compared the functional connectivity difference between the left and right PreCG between healthy controls and schizophrenia patients. Results show that the connectivity of the left Hippocampus with both left and right PreCG decreased in schizophrenia [Figure 3]. More details about the connectivity impairment of PreCG and the rest of the brain is demonstrated in the [Tables 2 and 3]. We also compared schizophrenia affects the PreCG in the male and female patients [Figures 4 and 5].

\section{DISCUSSION}

The present study supports the notion of the functional connectivity abnormal connectivity of the precentral gyrus in schizophrenia ${ }^{[1,2,8-11]}$. We have shown that PreCG alteration and its abnormal connectivity are not the 

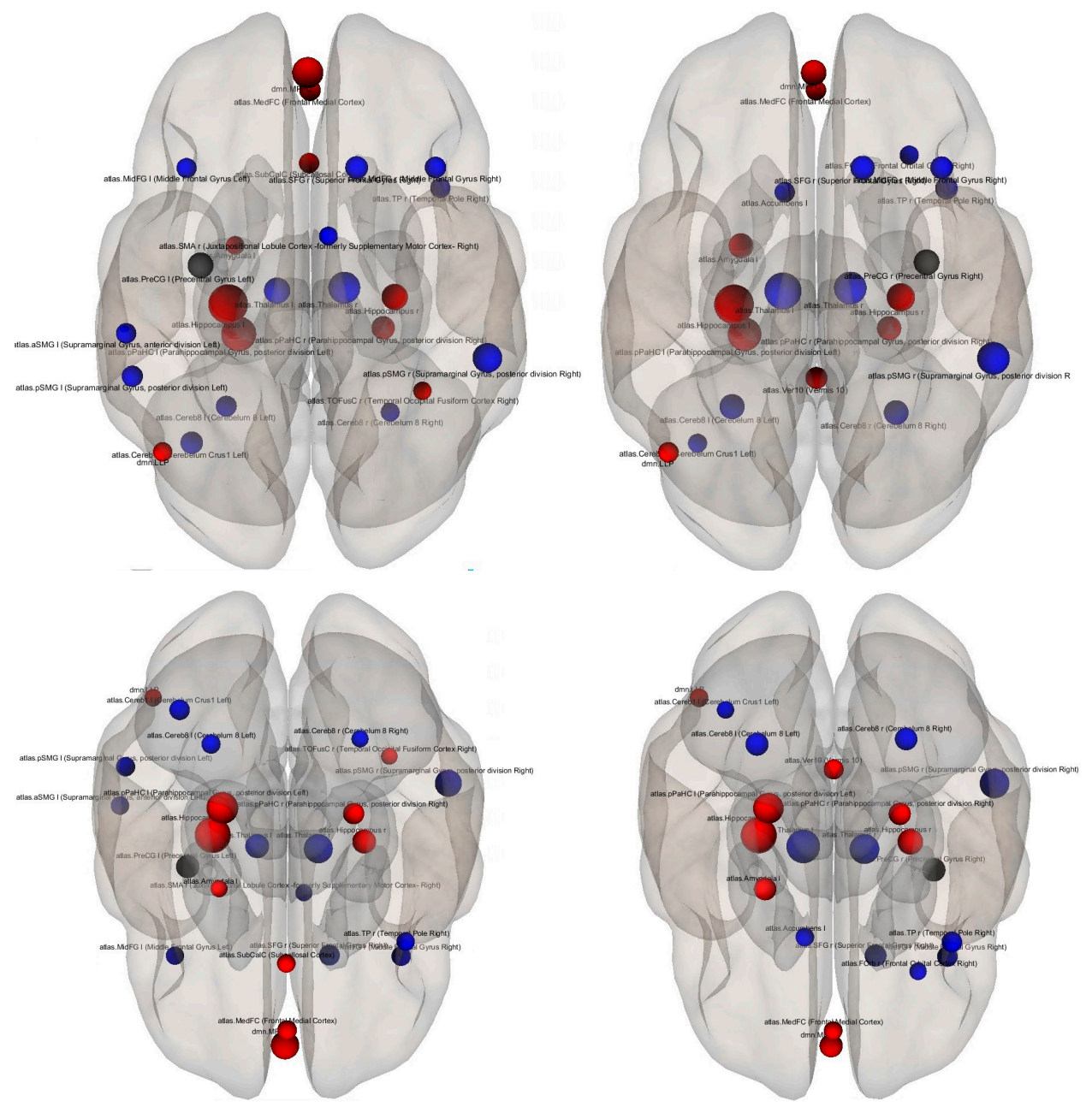

Figure 2. The superior (top) and the posterior views of the ROI-level results for each target ROI. The selected sources (precentral gyrus left and right) are shown by black dots and the targets are blue (control > schizophrenia) and red (control < schizophrenia). Effects size for each region is shown by the dot size

Table 3. The connectivity contrast values between the PerCG left and the targets $(P=0.05)$ (the complete list of the effects size is provided in the supplementary data)

\begin{tabular}{llll}
\hline Analysis Unit & \multicolumn{1}{c}{ Statistic } & p-unc & pFDR \\
\hline Seed intensity = 25.55, size= & PreCG I & $\mathrm{F}(30)(113)=3.41$ & 0.0000 \\
PreCG I -Hippocampus I & $\mathrm{T}(142)=4.47$ & 0.0000 & 0.0022 \\
PreCG I -pPaHC I & $\mathrm{T}(142)=4.02$ & 0.0001 & 0.0063 \\
PreCG I -Thalamus r & $\mathrm{T}(142)=-3.72$ & 0.0003 & 0.0128 \\
PreCG I -dmn.MPFC & $\mathrm{T}(142)=3.64$ & 0.0004 & 0.0131 \\
PreCG I -pSMG r & $\mathrm{T}(142)=-3.48$ & 0.0007 & 0.0179 \\
PreCG I -Hippocampus r & $\mathrm{T}(142)=3.12$ & 0.0022 & 0.0450 \\
PreCG I -Thalamus I & $\mathrm{T}(142)=-3.10$ & 0.0023 & 0.0450 \\
\hline
\end{tabular}

same in the male and female patients with schizophrenia. The resting-state functional magnetic resonance imaging data of healthy control subjects and patients with schizophrenia from the Centers of Biomedical Research Excellence dataset are used to examine the aberrant functional brain connectome in schizophrenia. Our results show precentral gyrus has abnormal communication with thalamus, hippocampus, pPaHC, pSMG and $\mathrm{mPFC}(\mathrm{pFDR}=0.05)$. 

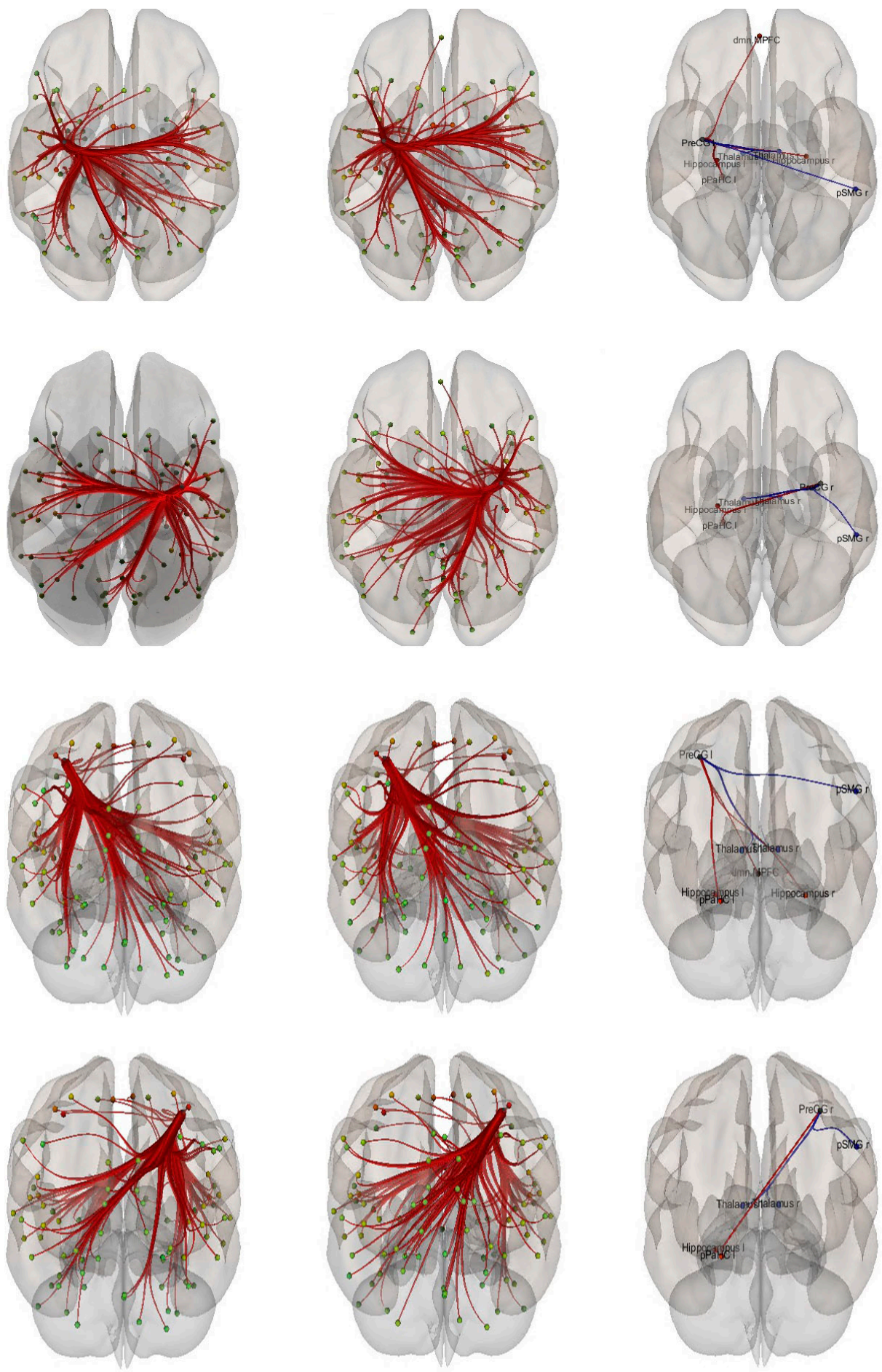

Figure 3. Functional connectivity difference between the left and right precentral gyrus between healthy controls vs. patients with schizophrenia (controls > schizophrenia). The PreCG connectivity in the healthy control, patients with schizophrenia, and their difference are shown in the first, second and the last column respectively and the two top rows are the superior views and the two-bottom row are the posterior views

Here, we investigated abnormal connectivities of left and right precentral gyrus in male and female patients with schizophrenia. The region of interest based analysis carried out for the healthy control and patients with Schizophrenia data. To do this resting state functional magnetic resonance imaging data of healthy control subjects and patients with schizophrenia from Center for Biomedical Research Excellence are used 

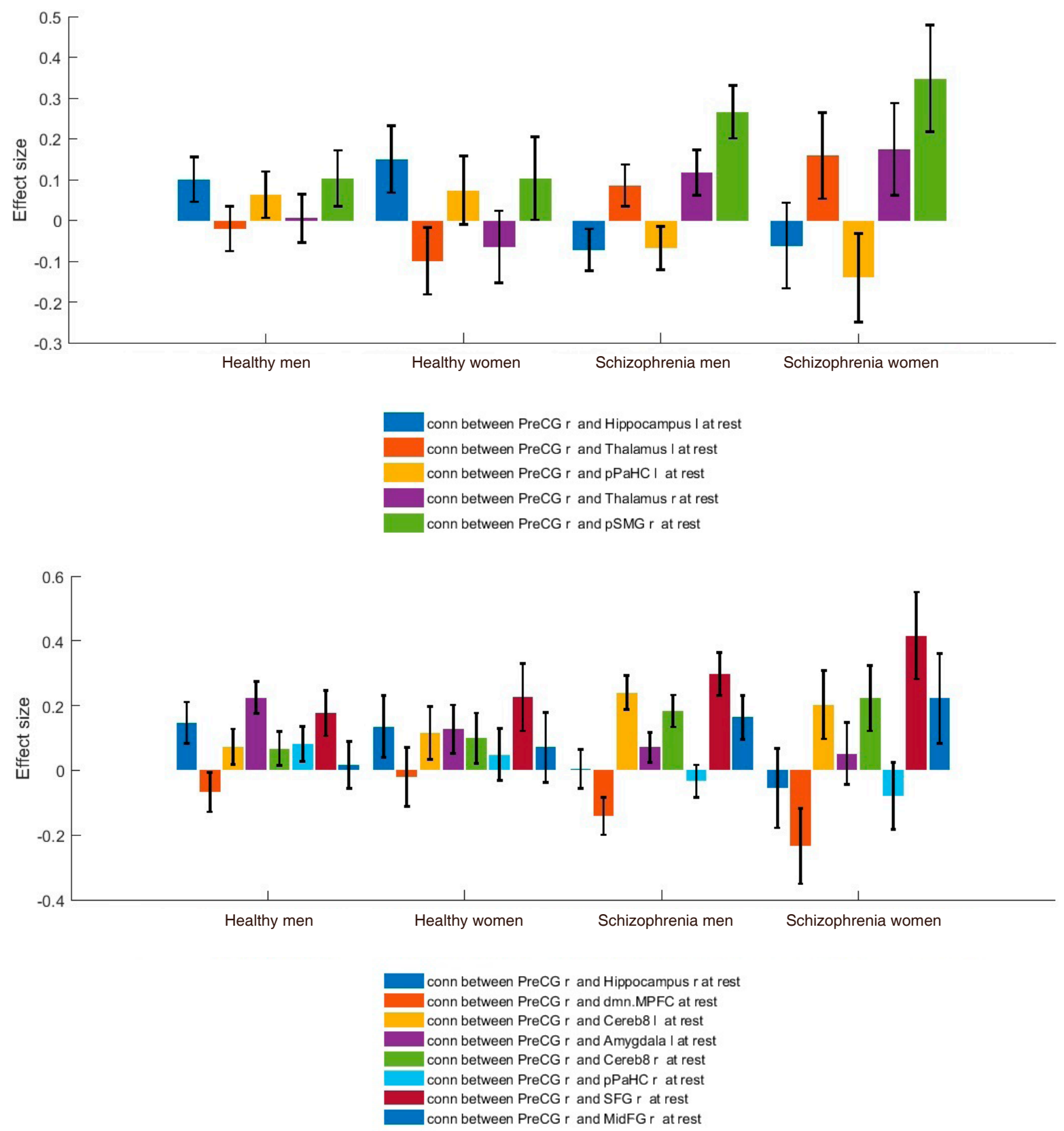

Figure 4. Right precentral gyrus functional connectivity difference between healthy controls and patients with schizophrenia (controls > schizophrenia)

to examine the aberrant functional brain connectome in schizophrenia which contains raw anatomical and functional MR data from 72 patients with schizophrenia and 75 healthy controls, ranging in age from 18 to 65 years old. We can summarize our results as follows: The precentral gyrus has abnormal communication with thalamus, hippocampus, pPaHC, pSMG and mPFC $(P=0.05)$. The right precentral gyrus has unusual connectivities with Hippocampus 1, pPaHC 1 Thalamus l, Thalamus $\mathrm{r}$ and pSMG $\mathrm{r}$. The right precentral gyrus has unbalanced connectivities with Hippocampus l, pPaHC l, Thalamus r, MPFC, pSMG r, Hippocampus $r$ and Thalamus 1 . The connectivity impairments of the PreCG in schizophrenia are different for male and female patients. This information is expected to provide a better understanding of altered functional connectivity of primary motor cortex in schizophrenia. 

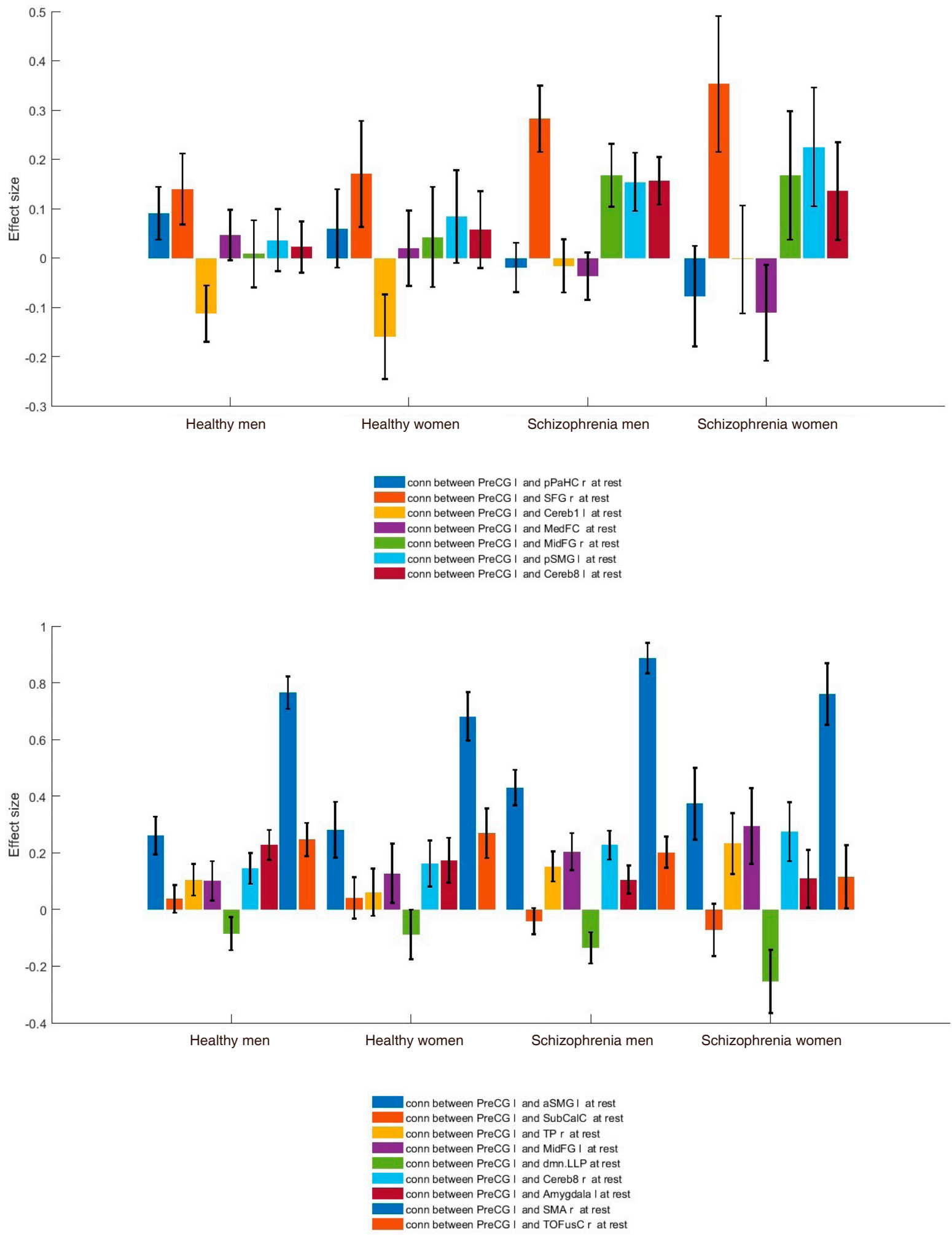


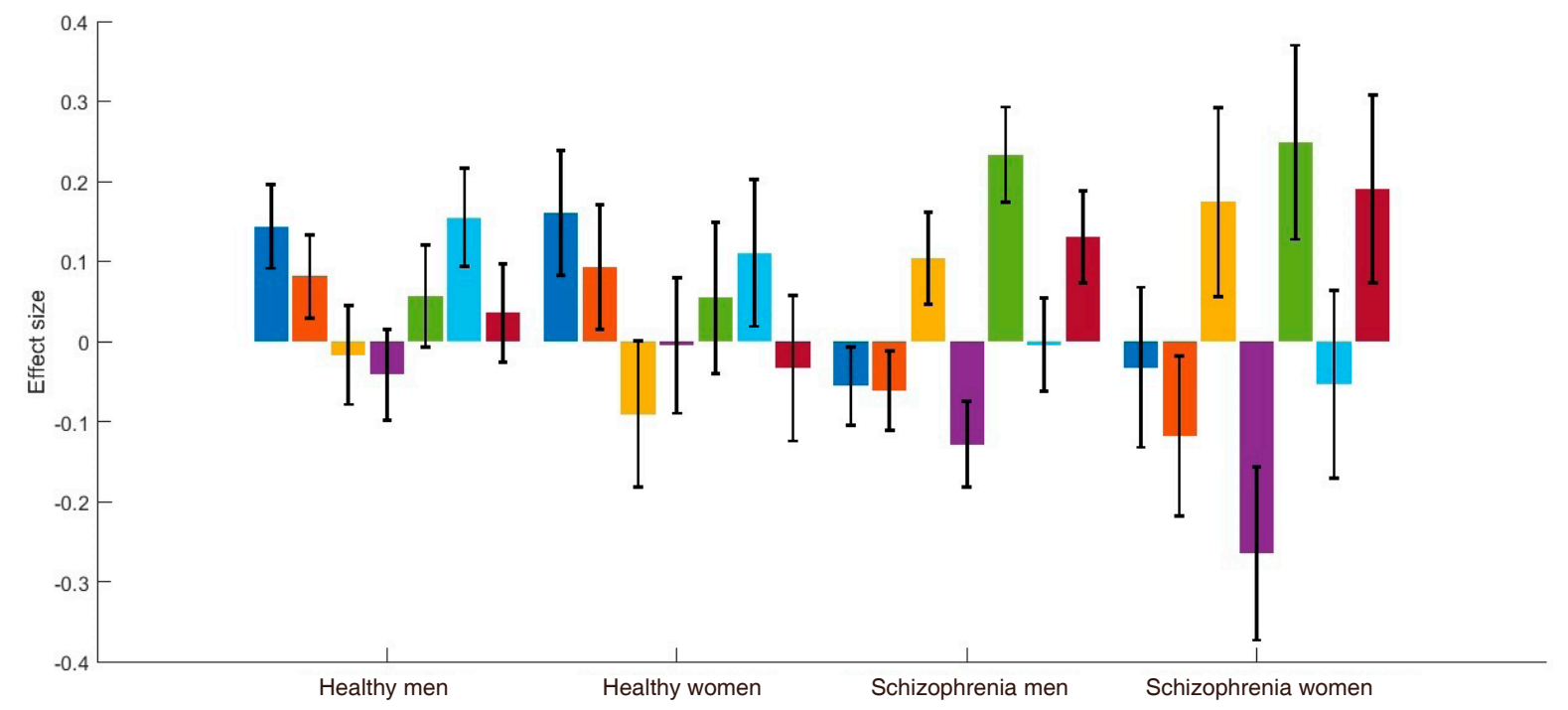

Figure 5. Left precentral gyrus functional connectivity difference between healthy controls and patients with schizophrenia (controls > schizophrenia)

\section{DECLARATIONS}

\section{Authors' contributions}

The author contributed solely to the paper.

\section{Data source and availability}

Datas presented here are from the Center for Biomedical Research Excellence data set (COBRE). This data set is available at a previous study ${ }^{[7]}$ and contains raw anatomical and functional MR data from patients with Schizophrenia and healthy controls, ranging in age from 18 to 65 years old. Resting fMRI, anatomical MRI, phenotype data for every participant including gender, age, handedness and diagnostic information are released.

\section{Financial support and sponsorship}

None.

\section{Conflicts of interest}

There are no conflicts of interest.

\section{Patient consent}

Not applicable.

\section{Ethics approval}

Not applicable.

\section{Copyright}

(c) The Author(s) 2018. 


\section{REFERENCES}

1. Hafner H, van der Heiden W. Epidemiology of schizophrenia. Can J Psychiatry 1997;42:139-51.

2. Howes OD, Murray RM. Schizophrenia: an integrated sociodevelopmental-cognitive model. Lancet 2014;383:1677-87.

3. Singh S, Goyal S, Modi S, Kumar P, Singh N, Bhatia T, Deshpande SN, Khushu S. Motor function deficits in schizophrenia: an fmri and vbm study. Neuroradiology 2014;56:413-22.

4. Mubarik A, Tohid H. Frontal lobe alterations in schizophrenia: a review. Trends Psychiatr Psychother 2016;38:198-206.

5. Shepherd AM, Quidé Y, Laurens KR, O’Reilly N, Rowland JE, Mitchell PB, Carr VJ, Green MJ. Shared intermediate phenotypes for schizophrenia and bipolar disorder: neuroanatomical features of subtypes distinguished by executive dysfunction. $J$ Psychiatr Neurosci 2015;40:58-68.

6. Li HJ, Chan CK, Gong QY, Liu Y, Liu SM, Shum D, Ma ZL. Facial emotion processing in patients with schizophrenia and their nonpsychotic siblings: a functional magnetic resonance imaging study. Schizophr Res 2012;134:143-50.

7. Xiao B, Wang S, Liu JB, Meng TT, He YQ, Luo XR. Abnormalities of localized connectivity in schizophrenia patients and their unaffected relatives: a meta-analysis of resting-state functional magnetic resonance imaging studies. Neuropsychiatr Dis Treat 2017; 13:467.

8. Poldrack RA. Region of interest analysis for fmri. Soc Cognit Affect Neurosci 2007;2:67.

9. Center for Biomedical Research Excellence data set (COBRE). Available from: http://fcon_1000.projects.nitrc.org/indi/retro/cobre. html [Last accessed on 12 Apr 2018]

10. Khadka S, Meda SA, Stevens MC, Glahn DC, Calhoun VD, Sweeney JA, Tamminga CA, Keshavan MS, O’Neil K, Schretlen D, Pearlson GD.Is aberrant functional connectivity a psychosis endophenotype? A resting state functional magnetic resonance imaging study. Biol Psychiatr 2013;74:458-66.

11. Bumseok J, Wible CG, Hashimoto RI, Kubicki M. Functional and anatomical connectivity abnormalities in left inferior frontal gyrus in schizophrenia. Hum Brain Mapp 2009;30:4138-51.

12. Lui S, Yao L, Xiao Y, Keedy SK, Reilly JL, Keefe RS, Tamminga CA, Keshavan MS, Pearlson GD, Gong Q, Sweeney JA. Restingstate brain function in schizophrenia and psychotic bipolar probands and their first-degree relatives. Psychol Med 2015;45:97-108.

13. ZaleskyA, Fornito A, Egan GF, Pantelis C, Bullmore ET. The relationship between regional and inter-regional functional connectivity deficits in schizophrenia. Hum Brain Mapp 2012;33:2535-49.

14. Mayer AR, Ruhl D, Merideth F, Ling J, Hanlon FM, Bustillo J, Cañive J. Functional imaging of the hemodynamic sensory gating response in schizophrenia. Hum Brain Mapp 2013;34:2302-12.

15. Stephen JM, Coffman BA, Jung RE, Bustillo JR, Aine CJ, Calhoun VD. Using joint ica to link function and structure using meg and dti in schizophrenia. Neuroimage 2013;83:418-30.

16. Calhoun VD, Sui J, Kiehl K, Turner J, Allen E, Pearlson G. Exploring the psychosis functional connectome: aberrant intrinsic networks in schizophrenia and bipolar disorder. Front Psychiatr 2011;2:75.

17. 143 adult subjects from COBRE dataset. Available from: http://fcon_1000.projects.nitrc.org/indi/retro/cobre.html [Last accessed on 12 Apr 2018]

18. Greve DN, FischlB. Accurate and robust brain image alignment using boundary- based registration. Neuroimage 2009;48:63-72.

19. Andersson JL, Jenkinson M, Smith S. Non-linear registration, aka Spatial normalisation FMRIB technical report TR07JA2. FMRIB Anal Group Univ Oxf 2007;3:1-21.

20. Rueckert D, Sonoda LI, Hayes C, Hill DLG, Leach MO, Hawkes DJ. Nonrigid registration using free-form deformations: application to breast MR images. IEEE Trans Med Imag 1999;18:712-21.

21. Gabrieli SW, Castanon AN. Conn: a functional connectivity toolbox for correlated and anticor related brain networks. Brain Connect 2012;2:125-41.

22. Behzadi Y, Restom K, Liau J, Liu T. A component based noise correction method (CompCor) for BOLD and perfusion based fMRI. Neuroimage 2007;37:90-101.

23. Peter McCullagh. Generalized linear models. Eur J Op Res 1984;16:285-92.

24. Friston KJ. Functional and effective connectivity in neuroimaging: a synthesis. Hum Brain Mapp 1994;2:56-78.

25. Deshpande G, LaConte S, Peltier S, Hu XP. Integrated local correlation: a new measure of local coherence in fmri data. Hum Brain Mapp 2009;30:13-23.

26. Goelman G. Radial correlation contrast-a functional connectivity mri contrast to map changes in local neuronal communication Neuroimage 2004;23:1432-9.

27. Kim JH, Lee JM, Jo HJ, Kim SH, Lee JH, Kim ST, Seo SW, Cox RW, Na DL, Kim SI, Saad ZS. Defining functional SMA and pre-SMA subregions in human MFC using resting state fMRI: functional connectivity-based parcellation method. Neuroimage 2010;49:2375-86.

28. Whitfield-Gabrieli S, Nieto-Castanon A. Conn: A functional connectivity toolbox for correlated and anti correlated brain networks. Brain Connect 2012;2:125-41. 